\title{
Le projet pluriactif entre autonomie et précarité. Les défis de l'accompagnement
}

Pluriactivity between autonomy and precariousness. The challenges of the accompaniment

Hélène Tallon et Jean-Philippe Tonneau

\section{OpenEdition}

Journals

Édition électronique

URL : http://journals.openedition.org/economierurale/3485

DOI : 10.4000/economierurale.3485

ISSN : 2105-2581

Éditeur

Société Française d'Économie Rurale (SFER)

Édition imprimée

Date de publication : 30 juillet 2012

Pagination : 42-55

ISSN : 0013-0559

Référence électronique

Hélène Tallon et Jean-Philippe Tonneau, « Le projet pluriactif entre autonomie et précarité. Les défis de l'accompagnement », Économie rurale [En ligne], 330-331 | juillet-septembre 2012, mis en ligne le 30 juillet 2014, consulté le 01 mai 2019. URL : http://journals.openedition.org/economierurale/3485 ; DOI : 10.4000/economierurale.3485 


\section{Le projet pluriactif entre autonomie et précarité Les défis de l'accompagnement}

Hélène TALLON, Jean-Philippe TONNEAU • CIRAD-ES, UMR Tétis, Campus International de Baillarguet htallon@gmail.com ; jean-philippe.tonneau@cirad.fr

\section{Introduction}

es crises sectorielles agricoles et la remise Len question de la société salariale, sur fond de nouvelle attractivité des territoires ruraux, se conjuguent pour former le cadre actuel du développement de la pluriactivité. Les espaces ruraux attirent de nouveaux migrants, qui sont à $61 \%$ des actifs dont $13 \%$ en situation " fragilisée » ${ }^{1}$ (MairieConseils, 2005). Ces espaces sont ainsi confrontés à une double problématique : celle de l'accueil d'actifs et celle du soutien à la création d'activité en contexte de faible offre d'emploi salarié. La question des projets pluriactifs et de leur accompagnement se pose frontalement (Boudy, 2009). En progression dans la création d'activité des néoruraux (Saleilles, 2007), ces systèmes d'activité peinent à trouver leur place dans les dispositifs d'accompagnement existants, du fait de leur précarité constitutive ou de leur distance au modèle normé de l'entreprise. Compte tenu de l'hétérogénéité des situations de pluriactivité rencontrées, et de la segmentation importante des dispositifs d'accompagnement, comment dès lors penser l'accompagnement à la pluriactivité ? Est-il possible de concevoir un accompagnement dépassant les cadres normatifs de l'entreprise pour valider des projets singuliers et contextuels ? Partant de l'hypothèse que pour être accompagné un

1. Personnes en difficulté en provenance d'une ville, personnes n'exerçant pas d'emploi, salariés saisonniers se sédentarisant, jeunes ou adultes en situation d'errance, gens du voyage en voie de sédentarisation. projet doit être reconnu, que cette reconnaissance passe au préalable par sa compréhension, nous avons cherché à caractériser les éléments structurants des projets pluriactifs (système économique de référence, ancrage territorial, motivations, gestion du temps, mobilité, etc.), et à concevoir les termes d'un accompagnement adapté à ces projets. Nous considérons en effet le dispositif d'accompagnement comme l'interface entre le territoire organisé et les activités développées par ses acteurs. La reconnaissance de la pluriactivité devient ainsi un enjeu territorial, dont l'accompagnement peut être le vecteur. Cette relation pluriactivité et accompagnement a été au cœur de la recherche, menée de 2007 à 2011, dans le cadre d'une thèse de doctorat en géographie (Tallon, 2011). Notre article présente de quelle manière la prise en compte de la pluriactivité influence les formes de l'accompagnement mais interroge aussi ses finalités. Nous avons organisé les résultats en trois parties. Une première définit la pluriactivité et ses besoins en accompagnement, besoins largement insatisfaits. Dans la seconde nous présentons l'expérimentation menée et les projets, en insistant sur les formes d'accompagnements testées et mises en œuvre. Une troisième partie analyse l'impact de l'expérimentation en soulignant que la pluriactivité que nous avons traitée, nécessite de nouveaux regards politiques, permettant de dépasser le cadre des statuts actuels. En conclusion, nous revenons sur la diversité des projets pluriactifs et sur l'importance de projets territoriaux qui reconnaissent et prennent en compte cette diversité. 


\section{Pluriactivité et accompagnement}

\section{La pluriactivité, des systèmes d'activité aux combinaisons multiples}

Le droit du travail définit la pluriactivité comme «l'exercice simultané ou successif par une même personne de plusieurs activités professionnelles différentes » (Casaux, 1993). Pour circonscrire cette définition trop large, nous parlerons de pluriactivité dès lors que les activités peuvent se pratiquer de manière indépendante et qu'elles correspondent à des filières, des formations ou des métiers distincts. Nous intégrons à notre définition le cumul de statuts sociaux (le salariat en particulier), dans la mesure où les cadres réglementaires des activités sont inféodés aux statuts et ont une incidence essentielle sur les stratégies de combinaison d'activité. En conséquence, pour analyser la pluriactivité, nous nous référons au concept de système d'activité. Pour Paul et al. (1994), «le système d'activité est un métasystème englobant l'ensemble des activités productives de la famille ». Ce concept est élargi par certains auteurs pour lui adjoindre l'ensemble des relations fonctionnelles entre les différentes sphères d'activité repérées, qu'elles soient marchandes ou non marchandes (Gaillard et Sourisseau, 2009). Pour ces auteurs, ce qui se joue dans ces relations peut expliquer la résilience d'agricultures économiquement et techniquement peu performantes. Parler de système d'activité pour désigner la pluriactivité permet ainsi d'y intégrer l'ensemble de ce qu'elle produit (revenu monétaire, lien social, identité, capacité d'échange etc.). Pour les auteurs cités, le système d'activité ne s'analyse qu'à l'échelle de la famille ou du ménage, entendus comme unité de production et de résidence, et caractérisés par le nombre de consommateurs qu'ils regroupent. La difficulté méthodologique à cerner le système d'activité est de fait importante. Les activités sont dans un premier temps appréhendées pour chaque individu, l'enjeu consistant dans un deuxième temps à mettre à jour et à considérer les liens reliant les activités des différents individus concernés. D'autre part, la création d'activité est bien souvent perçue comme une histoire personnelle. La dissociation des activités des membres du ménage est forte - y compris dans les exploitations agricoles - et l'accompagnement s'adresse majoritairement à un individu isolé. Nous verrons que, dans notre recherche, nous butterons sur la question de l'entité sociale à considérer pour cerner la cohérence des systèmes d'activité étudiés.

\section{Des politiques d'accompagnement à la pluriactivité plutôt ambiguës}

Les espaces ruraux sont, par choix ou par obligation, des espaces d'expérimentation de formes d'activités dites atypiques, informelles, alternatives, etc., parmi lesquelles figurent de nombreuses expériences de combinaisons d'activités professionnelles. La plupart d'entre elles tournent résolument le dos au modèle de protection sociale et de stabilité véhiculé par la société salariale. Elles traduisent une conception du travail qui s'appuie sur la valorisation de savoirs non académiques ou sur la mobilisation de compétences acquises tout au long du parcours professionnel mais aussi dans la sphère privée. L'utilité sociale du travail réalisé est aussi reconnue (Laurent et Mundler, 2006). Une relation forte entre lieu de vie et activité professionnelle caractérise ces systèmes (Lenain, et al., 2009). Elle induit un rapport spécifique au territoire, qui est d'avantage un cadre de vie, d'activité, et d'épanouissement du projet personnel, qu'un lieu producteur de ressource identifiée et évaluée pour une activité économique (Gumuchian et Pecqueur, 2007). De fait, dans les espaces ruraux éloignés de l'influence urbaine, la création et le fonctionnement du système d'activité pluriactif répond à des logiques plus existentielles qu'économiques (Saleilles, op. cit.). Cette constatation peut expliquer la dépendance fréquente des projets aux dispositifs d'aide 
sociale et au Revenu de solidarité active, et par conséquent, la peur des collectivités locales en charge de l'activité économique de voir la pluriactivité maintenir les populations dans la précarité. Les institutions sont peu à l'aise avec la pluriactivité.

Cependant, après avoir été combattue férocement : «la pluriactivité a longtemps été considérée comme une activité nécessaire de survie associant deux misères pour en faire une troisième » (Perret, 1999), la pluriactivité trouve aujourd'hui une certaine reconnaissance dans le tissu économique rural. Des politiques de soutien existent sur certains territoires (citons la mission agriruralité de la Région RhôneAlpes). Mais de manière paradoxale, la plupart des politiques de développement économique encouragent une pluriactivité exigeante en temps de travail et en compétences, en s'appuyant sur des statuts dont la souplesse est inversement proportionnelle à la protection qu'ils apportent. Ces réponses sont peu en phase avec la réalité économique des territoires fragilisés par la crise de la société salariale et posent la question de l'accompagnement des projets dans le contexte de dynamiques spécifiques à chaque espace rural. La sectorialisation des structures en charge de l'accompagnement ne facilite pas l'accompagnement de pratiques peu normées et par définition multisectorielles. D'autant plus que ces structures ont la lourde tâche d'apporter un appui à des projets parfois très instables, manquant de références techniques (qu'il faut souvent inventer), et dont les activités sont parfois très éloignées des modèles habituels.

Les dispositifs d'accompagnement à la mise en place d'une activité ont pour objectif d'aider le créateur à mener à bien son projet, dans les meilleures conditions possibles, en lui permettant de bénéficier d'un appui qui peut être technique, méthodologique, relationnel, et en l'introduisant dans le monde professionnel auquel il va s'identifier par la suite. L'accompagne- ment est un outil au service de l'émergence du projet. Si l'accompagnement a bien une fonction d'intégration ou de mise en convergence des activités vers les statuts existants, il est avant tout «une démarche visant à aider une personne à cheminer, à se construire, à atteindre ses buts » (Beauvais, 2004). D'un point de vue pratique, cette posture permet de coconstruire les problèmes avec les acteurs, et de prendre en compte des situations et des projets complexes. Le processus, continu mais non linéaire, fait de bifurcations et de doutes, laisse une plus grande ouverture à l'émergence des « possibles », même lorsqu'ils paraissent éloignés du point de départ. C'est ce postulat qui a été au fondement de la mise en œuvre de l'expérimentation que nous présentons ici.

\section{L'expérimentation}

\section{Une expérimentation entre recherche- action, formation et accompagnement}

Positionnée au cœur d'une géographie des pratiques, s'appuyant sur une posture constructiviste au sein de laquelle la réalité est sans cesse requestionnée par des individus « pluriels » et donnant leur forme à des rapports sociaux situés, la méthodologie qui forme l'armature de cette recherche découle d'emprunts à la sociologie et à l'anthropologie. Elle repose sur la combinaison d'une expérimentation et d'une recherche-action. L'expérimentation, intitulée "Formation/accompagnement à l'Entreprise rurale pluriactive », (ERP), a consisté à imaginer et à tester, à l'intérieur d'une formation à la création d'activité, de nouvelles formes d'accompagnement à la pluriactivité, et d'en analyser les effets. Une expérimentation a pour objectif de problématiser des situations dans lesquelles l'évidence des solutions se heurte aux limites instituées ou normatives (Debaise, 2005). Dans un processus de recherche, l'expérimentation ouvre des espaces de créativité, et permet 
d'agir «dans et contre la situation » (Nicolas-Le-Strat, 2007). Quant à la recherche, elle a été « action » car elle a mobilisé les acteurs de l'accompagnement dans la conception et la mise en œuvre de la formation. Le couplage recherche-action et expérimentation a permis une plus grande implication des chercheurs, et a valorisé les processus de connaissance permettant de passer du vécu empirique d'un noyau d'individus à une connaissance collective partagée de ce vécu. Expérimenter à l'intérieur d'une recherche-action offre donc a priori la possibilité de mettre à l'épreuve les dispositifs ou les situations mis en cause, et de faire bouger les lignes de force des cadres en place.

L'expérimentation a été menée dans le Haut-Languedoc héraultais. Dans cette montagne méridionale, la pluriactivité représente une part importante de l'activité du territoire, structurant non seulement les activités agricoles mais aussi l'ensemble des secteurs économiques (Tallon, 2005). Cette pluriactivité se développe dans un contexte économique difficile. Les cantons ruraux de l'Hérault sont les plus pauvres du département : revenus du travail faibles, taux de chômage et de RSA forts, installations de nouvelles populations sur un marché de l'emploi très restreint...

De janvier à octobre 2008, quatorze porteurs de projet ont participé à l'expérimentation, d'une durée de neuf mois si l'on ne prend en compte que la phase de présence quotidienne des porteurs de projet (avec le statut de «stagiaires de la formation professionnelle »), mais de plus de deux ans si l'on intègre la totalité de la démarche d'accompagnement. Le dispositif couplait modules de formation à la création d'activité, certains classiques (de l'idée au projet, le plan d'entreprise, etc.) et d'autres plus originaux (le territoire comme ressource pour le projet), aux modules d'accompagnement transversaux. Cette expérimentation a été menée par un groupe de chercheurs (Cirad, Université d'Avignon) et un ensemble de parte- naires : des structures d'accompagnement (Association Départementale Terres Vivantes $^{2}$, Ifad ${ }^{3}$, Ariès/Céméa ${ }^{4}$, Pays HautLanguedoc et Vignobles ${ }^{5}$ ), des formateurs et des porteurs de projet. La coordination du projet était assurée par le Cirad.

Sur les quatorze stagiaires entrés en formation, onze ont terminé le parcours. L'analyse porte donc sur onze projets, présentés comme pluriactifs en début de formation, c'est-à-dire combinant aux moins deux activités différentes. La moitié des porteurs de projet était au RMI (Revenu minimum d'insertion) ${ }^{6}$. Douze porteurs sur quatorze étaient des femmes (soulignons que les rmistes et les femmes sont les deux groupes ayant les plus fortes difficultés d'accès à l'emploi). Les porteurs avaient tous un parcours professionnel conséquent ; le niveau de formation initiale était hétérogène, de faible à très élevé. Tous disposaient de compétences pointues, dans le domaine de leur projet ou dans un autre domaine non réinvesti dans le projet. Seules deux personnes étaient issues du territoire. Pour les autres, l'installation pouvait être ancienne (plus de 20 ans) ou très récente (dans l'année). Une rapide description des projets permet d'apprécier la diversité des combinaisons d'activités, la moitié étant basée sur une activité agricole, et une partie combinant salariat et activité indépendante.

Les projets agricoles ont pour caractéristique commune d'être limités, en surface et en production. Quatre des cinq projets sont liés au maraîchage ou à la pépinière. Mathilde veut développer une pépinière de plantes aquatiques couplée à une activité de confection de bassins, la pépinière étant

2. Accompagne des porteurs de projets agricoles en installation progressive.

3. Accompagne les porteurs de projets artisanaux ou touristiques.

4. Accompagne les porteurs de projets artistiques. 5. Accompagne les porteurs de projets du territoire, sans distinction affichée de secteurs professionnels.

6. Devenu RSA en 2009. 
elle-même associée à de l'accueil et à de l'animation sur le lieu de production. Elle a 33 ans, et elle a exercé une activité de maraîchage biologique pendant 10 ans avec son ancien conjoint. Elle s'appuie sur cette compétence forte pour élaborer son projet. De la même façon, Lola, 49 ans, souhaite développer un projet de maraîchage lié à ses gîtes écologiques, pour accompagner son activité actuelle de création de bijoux. Gabrielle, originaire du territoire, a 55 ans. Elle expérimente une pépinière de tilleul truffier mycorhizé en parallèle à sa production de truffes. Elle entretien avec son mari, exploitant forestier, plusieurs hectares de chênes truffiers, dont la production est prévue pour les années à venir. Pour Colette, 48 ans, l'activité de maraîchage qu'elle souhaite mettre en place en parallèle à son activité de services à la personne (petit entretien des jardins, ménages) est tout à fait nouvelle. Elle dispose de terrains sur l'exploitation familiale, mais doit développer des compétences dans le domaine. L'activité de Nathalie est la seule activité agricole liée à l'élevage. Elle a 46 ans, et veut développer son élevage caprin, en lui couplant accueil et animation sur la bergerie. Elle exerce avec son mari cette activité d'élevage depuis plus de 10 ans, la difficulté étant de la faire passer à une échelle lui assurant une reconnaissance professionnelle et une stabilité économique.

Les autres projets intègrent largement services et formation. Ambre, 35 ans, veut développer une activité de yoga, couplée à une activité de thérapie, et à une troisième activité d'accompagnement à la naissance. Le yoga était l'activité professionnelle qu'elle exerçait en Angleterre, avant son retour récent en France. Laurent, 38 ans, souhaite combiner création de sites web et activité d'accueil et de séjours à thèmes, via les gîtes qu'il est en train de rénover. Graphiste de formation, il possède une solide expérience dans ce domaine, mais uniquement en Irlande, d'où il arrive tout juste. Carole, la compagne de Laurent, souhaite compléter le travail d'accueil dans les gîtes par des activités ponctuelles de formation ou d'expertise. À 55 ans, elle a de solides compétences, développées à l'étranger jusqu'à son installation récente dans le Haut-Languedoc. Frederica est comptable de métier. À 52 ans elle souhaite diversifier cette activité en proposant des formations et des prestations en comptabilité pour les très petites entreprises, tout en développant une production de plantes ornementales et potagères sur ses terrains. Brigitte, 53 ans, souhaite développer en parallèle à son activité d'accueil d'enfants en grandes difficultés sociales une activité artisanale de création de chapeaux et de travaux de couture. Agréée par le Conseil général et l'Aide sociale à l'enfance et fortement reconnue dans son métier, c'est pour s'extraire en partie de ce travail qui devient très fatiguant à son âge qu'elle se tourne vers une activité secondaire. Cécile, 39 ans, veut développer une activité d'animation autour de la capoeira tout en continuant son activité artisanale de fabrication de jeux de société. Elle non plus n'est pas novice dans cette dernière activité, ayant été artisan d'art pendant une dizaine d'années. Enfin Michelle, 51 ans, vend en ligne des partitions de musique anciennes et souhaite accueillir des cavaliers sur son terrain. Elle a de bonnes compétences en informatique, mais, comme Nathalie, elle est confrontée à la difficulté de faire passer son activité de vente à un niveau professionnel.

Pour terminer la liste, deux projets sont élaborés sur des compétences à confirmer ou à tester. Sarah, 33 ans, a comme projet d'ouvrir une boutique d'objets de décoration couplée à une activité artisanale de patine de meubles et de décoration intérieure. Sa formation de décoratrice d'intérieur est en cours, et elle n'a pas de compétence avérée dans le commerce. Ce projet est pour elle l'occasion de donner un cadre à ses démarches de formation. Éric, à 44 ans, a travaillé de nombreuses années dans l'hô- 
tellerie et le travail saisonnier agricole. C'est pour « se poser » qu'il souhaite développer une activité de reiki, technique de soin énergétique, pour laquelle il vient juste de se former.

Ces projets ont tous comme caractéristique d'être « hors norme », ou du moins posent-ils tous des questions à l'accompagnement, que ce soit par le modèle d'activité véhiculé, la capacité financière des porteurs, les compétences multiples à mobiliser, ou le statut adapté à ces combinaisons d'activité.

\section{Accompagner souplement et collectivement, dans un cadre temporel étendu}

L'expérimentation a cherché à s'adapter constamment à la complexité du projet pluriactif, ce qui s'est traduit par l'évolution constante de ses contenus. C'est le développement des compétences transversales et plus particulièrement celles liées à la créativité, à l'ouverture et à la capacité de construire et de formaliser une stratégie, qui a été recherché. L'expérimentation visait à rendre les porteurs autonomes, leur permettant d'affirmer et de défendre leurs choix, pour in fine maîtriser leurs projets. Comme nous l'avons vu, l'expérimentation a alterné accompagnement à la construction du projet pluriactif et modules de formation permettant l'acquisition de compétences pour la conduite d'une activité pluriactive. Les phases de formation, présentées cidessous, ne doivent pas être comprises comme des étapes successives et temporellement balisées mais comme une imbrication de démarches co-construites au fil du processus.

La première phase a consisté à mettre en place le cadre de transformation de l'idée en projet. Bien entendu le choix de travailler sur un « projet » n'est pas neutre. Formant le socle des démarches actuelles de formation et d'apprentissage, l'injonction au projet apparaît comme une nouvelle contrainte sociale (Jaillet, 2002). Le projet est en effet la base de l'engagement du travailleur modèle du nouveau capitalisme : «Les personnes ne feront plus carrière mais passeront d'un projet à l'autre, leur réussite sur un projet donné leur permettant d'accéder à d'autres projets plus intéressants » (Boltanski et Chiapello, 1999). Il est aussi un élément régulateur entre d'une part l'individu aux prises avec les incertitudes grandissantes provoquées par les transformations du travail et d'autre part les politiques publiques de moins en moins protectrices et stabilisatrices. L'individu devient le seul responsable de son devenir. Il ne lui suffit plus de se chercher une place mais il doit la construire : "Dans le contexte de la société postindustrielle marquée par le flou, l'aléatoire et l'incertain, les projets doivent être impérativement déclinés pour que l'individu justifie sa légitimité à exister» (Boutinet, 2002). Mais au-delà de son injonction normative, c'est dans sa capacité à mobiliser les acteurs et à transformer une idée en « réalisable » que la notion de projet a été utilisée dans l'expérimentation. En effet, pour chaque personne, le projet représentait un futur possible et désirable à court terme. C'est bien dans ce sens que l'expression «porteur de projet »a été employée pour désigner les pluriactifs de l'expérimentation, individus cheminants dans leur propre devenir professionnel. Le travail des deux premiers mois, en impliquant le passé du porteur en tant qu'élément de réorganisation et de dynamisation du projet ( ibid) et en mettant en évidence les bifurcations constitutives des parcours de vie, a considérablement déstabilisé les porteurs, montrant par là même que le projet ne pouvait être pris en compte que dans son contexte et en référence à eux-mêmes.

Au bout de ces deux mois, les porteurs ont été invités à présenter leurs projets à un groupe de personnes, représentants de collectivités locales et de structures d'accompagnement. C'est à cette occasion que les porteurs ont rencontré pour la première fois les structures d'accompagnement qui chemineront par la suite à leurs côtés. Très rapidement cette présentation a été conflic- 
tuelle. Les porteurs ne se reconnaissaient ni dans la vision de l'activité indépendante défendue par certaines structures d'accompagnement, ni dans l'appellation d' « entrepreneur ». D'autre part, les incohérences et les difficultés relevées dans certains projets ont placé les accompagnateurs dans une position d'évaluateur qui n'avait pas lieu d'être à ce stade. Ce conflit a débouché pour les porteurs sur une crise de confiance, et pour les accompagnateurs sur une difficulté à s'engager. Cette crise a été résolue par un travail de fond qui a réaffirmé les grands principes de l'accompagnement : cheminement « avec» le porteur, coconstruction du projet, aller-retour entre les intentions, les changements et le but attendu, évaluation par le porteur, à l'intérieur d'un espace de dialogue "pacifié ».

La deuxième phase, la structuration et la mise en perspective du projet dans son environnement (le territoire du projet), a permis au porteur de confronter son projet avec sa réalité économique et sociale, et d'intégrer les règles de gestion d'une activité indépendante. L'exploration des «possibles » qui s'offraient au porteur a été rendue plus concrète par la visite de structures pluriactives sur le territoire. Le territoire dans ses diverses dimensions a été longuement questionné, donnant lieu à l'élaboration de cartes de projets, qui se sont avérées dans plusieurs cas de véritables outils d'aide à la décision. Ambre a ainsi choisi un autre territoire d'implantation plus en phase avec les ressources nécessaires à son projet : à proximité d'une grande ville et de son réseau d'appui relationnel et familial. D'autres porteurs ont réorienté leurs stratégies de mobilité. Certaines cartes ont en effet révélé l'éclatement spatial des activités, et la fragilité du projet compte tenu de logiques spatiales contradictoires. L'espace a été aussi un moyen d'interroger la viabilité du projet quant à son organisation dans le temps : les mobilités régulières et lointaines imposées par l'une ou l'autre des activités peuvent ne pas avoir d'incidence négative sur le projet si elles ne sont pas simultanées. Par contre, des contraintes de présence simultanées sur deux lieux, même proches, peuvent entraver sérieusement le projet. Plus largement, ces cartes témoignent fortement des représentations que se fait le porteur de son espace de vie, et des contradictions existantes entre le désir de vivre dans un territoire et ses implications, lourdes, en termes de déplacements. Au cours de l'expérimentation, la compréhension du rôle joué par les distances et les contraintes logistiques dans l'organisation de la pluriactivité a ainsi constitué une grille de lecture pertinente pour comprendre les projets et leur faisabilité (Tallon, et al., 2009). À la suite de ce constat, cette grille a été mobilisée en tant qu'outil d'accompagnement.

La troisième phase, la traduction du projet en prévisionnel d'activité, avait bien entendu comme objectif de passer du cadre virtuel du projet à un cadre beaucoup plus contraignant passant nécessairement par l'évaluation du projet. Bien que l'évaluation financière soit dans le cadre de l'accompagnement une étape obligée, dans l'expérimentation elle n'a pas été centrale. Elle n'est apparue qu'en fin de processus, après la mise en place de l'ensemble des cadres permettant au porteur d'évaluer son projet de son propre point de vue (faire sens, valoriser ses compétences, avoir une visibilité et être reconnu, valoriser une innovation, etc.). C'est au vu des attentes formulées par le porteur que seront analysés les aspects économiques du projet. Le prévisionnel a été réalisé par la méthode du «calcul du seuil de rentabilité », méthode qui met en perspective la rémunération minimale souhaitée par le porteur avec le chiffre d'affaires à réaliser et la quantité de travail nécessaire pour atteindre cet objectif. Malgré une rémunération attendue faible (huit personnes sur quatorze attendaient moins d'un smic de leur projet), les prévisions de résultats financiers des projets ont été, à l'exception de deux projets, largement déficitaires. Ces résultats ont, dans un premier temps, remis 
en cause la viabilité du projet. Ce blocage a amené les pluriactifs et leurs accompagnateurs à analyser différemment les projets. La manière de calculer le prévisionnel ne prenait pas en compte l'ensemble du système d'activité : l'activité salariale, quand elle existait, ou les revenus familiaux, n'étaient pas intégrés dans le calcul réalisé, et le recours aux formes de mutualisation ou d'échange n'était pas valorisé. Or ces deux éléments (inscription du projet dans un système d'activité dont l'entité sociale doit être définie avec précision ${ }^{7}$ et échanges ou inscription dans des collectifs d'activité) ont été identifiés par les porteurs comme des éléments de réponse possible aux difficultés d'intégration de leurs pluriactivités au cadre classique de l'entreprise.

\section{Quatre modalités d'accompagnement complémentaires}

Parallèlement aux trois phases décrites, s'est déroulé le processus d'accompagnement. L'accompagnement a été réalisé par sept structures de natures différentes : trois structures d'accompagnement proprement dites (ADTV, Ifad, Ariès), deux organismes de recherche (Cirad et Université d'Avignon), une collectivité territoriale (Pays HLV), une organisation professionnelle agricole (Mutualité Sociale Agricole). L'accompagnement a suivi quatre modalités, qui ont fonctionné en interaction les unes avec les autres.

La première modalité a été de proposer un accompagnement individuel « classique ». Le porteur était seul avec l'accompagnateur, qui appliquait la méthodologie de sa structure d'accompagnement. Le porteur a été suivi par cet accompagnateur tout au long de l'expérimentation. Deux innovations peuvent être relevées

7. Il existe sur le territoire des exemples de collectifs d'activité, qui interrogent cette entité sociale. De plus, la recomposition des familles oblige à repenser finement le cadre d'échange familial qui conditionne le système. par rapport à l'accompagnement prescrit dans le cadre des parcours à la création d'activité : le porteur avait le choix de la structure qui l'accompagnait, et il pouvait passer d'une structure à une autre, de manière à explorer les différentes facettes de sa pluriactivité. Ce choix était cependant relatif car largement conditionné par les compétences de chaque structure. En théorie ce choix existe aussi dans le cas d'une prescription. Mais il est limité dans la pratique par les conventions liant les organismes au statut du porteur de projet. Les structures d'accompagnement impliquées dans cette première « forme » étaient celles vers lesquelles le porteur aurait été orienté dans le cas d'un parcours prescrit (associations intervenant sur le territoire). Le bilan de cet accompagnement a été mitigé. Du fait d'interventions parfois trop courtes, certains porteurs ont eu le sentiment de ne pas être écoutés. D'autres n'ont pas compris comment mettre en pratique les conseils non coordonnés qui leur étaient donnés par des structures différentes. L'offre d'accompagnement était finalement trop limitée (trois structures). Seuls les porteurs ayant un projet agricole, déjà bien construit et disposant de la majeure partie des ressources nécessaires, ont été satisfaits de cette forme d'accompagnement et continuent à y faire appel après la formation. Cinq personnes sur quatorze sont dans ce cas.

Dans la deuxième modalité, l'accompagnement a été réalisé au sein d'un collectif élargi. Chaque porteur de projet s'est trouvé face à une structure d'accompagnement et aux autres membres du groupe de l'ERP. Les questions relatives à chaque projet ont été discutées en commun. L'ensemble du groupe participait aux échanges et a pu apporter son expérience au projet concerné, tout en profitant dans le même temps des informations qui circulaient. Ces interventions, très appréciés des porteurs, ont été répétées plusieurs fois, à leur demande. 
Nous appelons ces échanges de groupe « accompagnement » car l'intervention avait bien pour but de coconstruire les projets individuels. La mutualisation des compétences et des expériences de chacun a été d'un apport essentiel aux projets individuels. Elle a permis de répondre à des questions ou remarques très spécifiques, et a révélé les nombreux regards qu'il était possible de porter sur un même projet. De manière plus inattendue, l'implication des « pairs », dans ce cas les autres porteurs, a souvent contribué à valider le point de vue de l'accompagnateur, qui pouvait ainsi rester dans une position souple, laissant la place à ses propres incertitudes car il était secondé par le groupe dont il sollicitait l'expérience et le point de vue.

La troisième modalité, l'accompagnement individuel par un collectif de structures d'accompagnement, a été testée à la fin de l'expérimentation. Elle n'avait pas été envisagée au départ, mais le constat des lacunes de l'accompagnement en face à face et la difficulté des porteurs de projet à se « situer » parmi les divers points de vue, conseils ou informations apportés par les différentes structures rencontrées, a conduit le comité de pilotage de l'ERP à repenser cet accompagnement. Le face à face avec le porteur est fondamental, et l'accompagnement en groupe ne s'y substitue pas. Mais il a vite montré ses limites dans l'expérimentation : en situation de pluriactivité, le porteur doit croiser des éléments concernant plusieurs activités pour mettre en place une stratégie d'ensemble. Il est donc amené à collecter des points de vue issus d'accompagnateurs de différentes structures. Or, même à l'intérieur d'une même structure, les vues peuvent diverger. Les porteurs se sont trouvés confrontés à la question de la pertinence de l'information et aux critères de choix de celle-ci. Dans cette forme d'accompagnement, qui réunit plusieurs accompagnateurs autour d'un porteur de projet, ce sont les compétences et les expériences des accompagnateurs qui sont d'abord mutualisées. Sont aussi mutualisées les questions, et en particulier celles en suspens. Ces questions changent alors de statut : de simples doutes ou sentiments d'incompréhension, elles deviennent des enjeux de l'accompagnement à la pluriactivité.

La quatrième et dernière modalité est celle qui a concerné l'accompagnement du projet collectif. Dès le début de l'expérimentation, il est apparu très clairement que les projets comme les porteurs entraient mal dans le cadre classique de la création d'activité. Une seule personne se projetait dans une démarche «entrepreneuriale » ${ }^{8}$. Les assises matérielles des projets étaient faibles ou inexistantes, les combinaisons d'activité singulières et innovantes, les compétences peu mises en valeur. Face à ces limites, à l'inverse d'une vision entrepreneuriale, les porteurs défendaient des critères liés à une qualité de vie ou à un choix d'autonomie et de petit revenu, et recherchaient à travers leur projet, plus qu'une reconnaissance professionnelle, une reconnaissance de leurs valeurs. Ils se situaient principalement dans un registre de motivation centré sur les valeurs de solidarité, de partage ou d'échange. L'expérimentation a fait apparaître le rôle clef des démarches collectives, la viabilité de certains projets leur étant totalement conditionnée. Porteurs et accompagnateurs ont émis l'hypothèse qu'un projet collectif pouvait aider des projets individuels à émerger et à se développer. Tout au long de l'expérimentation a été conduite une réflexion sur ce que pourrait être un projet collectif fédérateur. Ses objectifs, sa mise en œuvre et sa dynamique ont été longuement discutés, et plusieurs ébauches ont été envisagées. La réflexion a débouché, en fin d'ERP, sur la mise en place d'une association des pluriactifs. Cette démarche

8. Terme qui n'a d'ailleurs jamais été défini dans l'expérimentation. 
collective a renforcé le projet individuel, les porteurs se sentant investis et rassurés quant à l'appui du groupe après la formation.

\section{Une démarche d'accompagnement pertinente mais une faible reconnaissance du projet pluriactif}

La combinaison des quatre formes d'accompagnement, coconstruites au cours de l'expérimentation (excepté la première), a permis de cheminer, non sans heurts mais en arrivant au bout du processus escompté. L'accompagnement a répondu aux incertitudes apportées par la complexité des systèmes d'activité pluriactifs par une réorientation permanente de sa pratique et de la prise en compte des besoins émergents : la prise en compte du territoire, en tant que ressource pour le projet et espace de reconnaissance du projet ou de l'individu, la valorisation de l'expérience (des porteurs et des accompagnateurs) permettant de relativiser les savoirs et les pratiques, la projection technique et financière en l'absence de références directement mobilisables, et l'adhésion côté accompagnateur comme côté porteur, à une démarche d'écoute attentive, non normative, ouverte sur l'incertitude et le doute, malgré les difficultés de projection que cela peut entraîner pour l'accompagnateur qui, même s'il chemine « avec » (Paul, 2004), reste dans une position de soutien. L'expérimentation montre la relation de réciprocité entre accompagnement et projet : les deux interagissent, à la condition impérative, comme nous avons pu le constater, que la souplesse d'adaptation de l'un comme de l'autre soit préservée.

Le succès de l'expérimentation s'exprime sans conteste dans la capacité que les porteurs ont pu acquérir à argumenter et à défendre leurs projets (aspect relevé par dix personnes sur onze). Portés presque uniquement par des femmes, les projets étaient pour elles une voie d'affirmation et de reconnaissance de choix de vie. Mais au-delà du développement de cette compétence, l'ex- périmentation a-t-elle aidé à construire des projets opérationnels ? Ce point est essentiel, et il faut ici différencier la perception des mandataires de celle des acteurs impliqués. Malgré tous les discours sur la place et l'importance du projet dans la société et son rôle dans l'épanouissement personnel et professionnel, la validation d'un projet par les pouvoirs publics repose principalement sur le critère de sortie du RSA ou du chômage. La prise en compte de l'environnement du projet atténue en partie cette position. C'est ainsi qu'un projet qui permet l'accès au régime de cotisant de solidarité (dans le cas d'une activité agricole) peut être reconnu par la MSA. Idem pour un projet qui apporte à la femme, dans un projet de couple, un statut, même annexe. Mais au fond ces considérations ont peu de poids face à l'injonction d'autonomie financière. Cela explique le malaise de l'accompagnement face aux motivations existentielles, politiques, sociales ou identitaires sur lesquelles sont bâtis certains projets de l'expérimentation.

En définitive, selon ces critères, bien peu de projets sont une «réussite ». À la fin des neuf mois de l'ERP, seul un projet est en place. Faut-il en rester à ce constat ? Comment valoriser la richesse du cheminement réalisé dans l'accompagnement? La question de la temporalité de la mise en place du projet, de la maturation de l'idée et de sa traduction opérationnelle en passant par les phases de test nécessaires, est très variable et peut être longue. Par exemple, en début d'ERP aucun porteur ayant un projet agricole n'avait le foncier suffisant pour mettre en place son activité. L'expérimentation a permis aux personnes d'élargir leurs réseaux et d'affiner leurs stratégies d'installation, leur donnant ainsi plus ou moins directement accès à des terres : Nathalie a loué des parcours communaux pour ses chèvres, négociés après présentation du projet à la mairie, tandis que Mathilde a négocié un bail sur le terrain maraîcher qu'elle avait en vue, par l'intermédiaire des 
accompagnateurs de l'ERP. La question de la qualification du projet est elle aussi importante. La posture constructiviste de l'accompagnement amène à une validation par le porteur lui-même, qui, comme on s'en doute, diverge des validations par les institutions ou par la sphère sociale et professionnelle. Elle repose pour le porteur sur la perception d'un progrès et des contraintes à lever. Frédérika, très fortement opposée à toute forme de salariat, a, petit à petit, élaboré sa combinaison d'activité autour d'une activité salariée qui permet de faire vivre le projet. Colette peut, à la fin de l'ERP, revoir à la baisse son projet de maraîchage car elle ne le mesure plus en termes de chiffre d'affaires mais en termes d'inscription dans le patrimoine familial. La perception des contraintes du projet a poussé le groupe à envisager des solutions portant sur d'autres statuts et sur des revendications collectives. C'est ainsi que les projets individuels ont été englobés dans des actions collectives plus larges : en sortie de formation, les porteurs ont créé une association, Les pluriactifs des Hauts-Cantons, permettant de disposer d'un espace très ouvert de conseil et de veille sur la pluriactivité. L'association a depuis sa création mené un certain nombre d'actions sur le territoire : participation collective à des marchés paysans (un stand commun sous une bannière « pluriactivité »), organisation de journées d'information publiques autour de la pluriactivité, lancement d'un questionnaire pour connaître les besoins de la population en termes de création d'activité, prévision d'organisation de journées avec des accompagnateurs, etc. Espace d'échange, d'information et de réflexion, l'association permet aux pluriactifs de la formation de rester étroitement en contact et leur apporte une reconnaissance locale : cette association reçoit le soutien (assez effacé certes) des institutions locales (prêt de salles, encouragements...) et l'appui des structures d'accompagnement présentes dans la formation. Cette action collective rend visible sur le territoire une problématique très souterraine et fait émerger des créateurs d'activité jusqu'alors assez effacés et peu impliqués dans les actions institutionnelles du territoire. D'autres actions collectives plus ciblées ont été mises en place : ouverture d'un atelier commun, échanges de services, etc. Ces dimensions collectives recouvrent des pratiques dont les finalités sont diverses : diminution des frais liés à l'activité par la mutualisation et les échanges, création de lien et de réseau, reconnaissance sociale, affirmation d'une identité professionnelle, etc. Cependant, à l'épreuve du temps et de la montée en puissance des projets individuels, ces démarches collectives s'affaiblissent ou changent de nature. Deux ans après sa création, l'association des pluriactifs n'organise plus d'évènements, alors que les échanges de services entre membres de l'ERP sont toujours actifs.

Notre expérimentation met en exergue le « système d'incertitudes » dans lequel gravitent les projets pluriactifs. Ce dernier rend difficile non seulement leur accompagnement, mais surtout la reconnaissance de leur place à part entière dans la structuration économique d'un territoire. De plus, «il apparaît clairement que si c'est la mise en ouvre elle-même d'un projet qui crée les conditions de possibilité de sortir de l'engrenage des statuts précaires, l'engagement dans un projet ne se décrète pas » (Leclerc-Olive et Engrand, 2000). Ces observations questionnent l'injonction au projet qui a trouvé ses limites chez plusieurs porteurs de la formation et la capacité de la démarche d'accompagnement à pallier les vides créés par les mutations actuelles du travail et des systèmes sociaux qui lui sont liés. Les participants à l'expérimentation revendiquent par leur projet la possibilité de sortir des statuts de dépendance dans lesquels ils sont à l'entrée en formation (chômage, RMI, mais aussi dépendance au revenu du mari, les femmes occupant une place particulièrement importante dans cette expérimentation) et mènent un combat poli- 
tique par leur approche du travail. Elles questionnent aussi la validation du projet par les institutions et les professionnels du territoire, voire par la société lorsque telle est la visée du projet. Plus le projet sera lié à une personne ou un choix de vie, plus les activités seront singulières, le lien entre elles instable, la référence à une profession et à un statut brouillés, plus le projet aura du mal à être reconnu. L'accompagnement a alors un rôle d'interface important à jouer. Cependant, au-delà de la validation du projet, c'est la validation même de la démarche de pluriactivité qui est en question. En effet les stratégies d'accompagnement sont très diverses selon la justification de la pluriactivité promue par les organisations : adaptation des conditions de vie et de travail des personnes concernées pour pérenniser les situations de pluriactivité, encouragement, voire accompagnement vers un retour à la monoactivité en considérant la pluriactivité comme une situation transitoire, construction d'une situation administrative de monoactivité pour permettre aux pluriactifs de bénéficier de droits liés à un statut social, enfin reconnaissance sociale de la polyvalence et de la mobilité professionnelle des individus par la mise en place d'un statut adapté à la souplesse de l'activité (Laurent et Mundler, op. cit.).

Certes l'expérimentation montre qu'il est possible d'adapter la démarche d'accompagnement aux projets pluriactifs, lorsque ses cadres peuvent rester souples et que ses espaces de régulation réflexifs et non normatifs sont actifs. Mais l'expérimentation montre aussi que le dispositif d'accompagnement, en tant qu'espace de reconnaissance et de validation des projets, est fortement lié aux politiques institutionnelles, qui agissent à différents niveaux sur un territoire. Les questions posées à l'accompagnement de la pluriactivité sont ainsi avant tout des questions posées à la société, par le biais du territoire. La question de la précarité, ou de l'adaptation à la faiblesse du marché du travail, ou plus largement la question de l'économie locale sur un territoire donné, ne sont que les éléments englobants des projets pluriactifs de l'ERP avec lesquels il faut composer.

\section{Conclusion}

Au cours de l'expérimentation, les projets ont tous suivi une démarche similaire : recherche d'une visibilité professionnelle et d'une existence légale en adaptant les statuts disponibles au projet, revendication d'un choix assumé de petit revenu, qui oblige à repenser l'activité indépendante en dehors des formes classiques d'entreprenariat, et recherche d'autonomie, celle-ci étant apportée par l'autoconsommation, l'auto construction, les échanges, la faible consommation d'énergie, l'emprunt réduit, voire nul. Petit revenu n'est pas lié à petit projet dans l'esprit du porteur. L'expérimentation menée dans le Haut-Languedoc a mis en évidence que la traduction en projet d'une démarche d'activité incluant de nombreuses facettes ne va pas de soi. L'inscription dans un cheminement balisé, dont l'objectif est de valider la pertinence d'une stratégie de rémunération de l'individu, pose problème lorsque les références n'existent pas, lorsque le porteur fait le choix de s'extraire d'un modèle qui lui paraît loin des valeurs qu'il veut défendre, et que la flexibilité des stratégies de combinaison d'activités est parfois le gage de survie de l'activité. Il est d'autre part difficile de repérer au sein de ces pratiques des éléments généralisables qui pourraient servir de base à la construction de dispositifs d'appui et de soutien à la pluriactivité. La singularité des situations rencontrées, la référence à un projet de vie, les difficultés de combiner les statuts sont autant de situations non reproductibles et qui nécessitent une attention spécifique de la part de l'accompagnateur.

Dans l'expérimentation ERP, les balbutiements et les maladresses de l'accompagnement n'ont été que l'expression de différentes visions des finalités d'un projet 
pluriactif. Enfin, les dispositifs, ou lieux d'élaborations de "petits arrangements ponctuels dans le temps et dans l'espace» (Beuret, 1999) résultent de rapports de force et de compromis territoriaux qui contraignent le jeu des acteurs et assignent à chacun une place déterminée. Or certaines formes de pluriactivité, qu'on observe dans le HautLanguedoc mais aussi au-delà dans l'ensemble des territoires ruraux, n'ont pas de cadre structurel d'expression, qu'elle soit de l'ordre de l'identité, de la revendication ou de la reconnaissance professionnelle. L'espace d'accompagnement ne peut à lui seul jouer ce rôle. Accompagner la pluriactivité demande plus que la simple adaptation des démarches d'accompagnement. Il s'agit d'un projet de développement territorial qui implique un positionnement politique affirmé, appuyé sur la connaissance fine du fonctionnement des activités d'un territoire, et sur la reconnaissance de l'apport de la pluriactivité dans sa dynamique économique et sociale.

\section{RÉFÉRENCES BIBLIOGRAPHIQUES}

Beauvais M. (2004). Des principes éthiques pour une philosophie de l'accompagnement. Savoirs, $\mathrm{n}^{\circ}$ 6, p. 99-109.

Beuret J.-E. (1999). Petits arrangements entre acteurs... Les voies d'une gestion concertée de l'espace rural. Nature, science et société, vol. 7, $\mathrm{n}^{\circ}$ 1, p. 21-30.

Boltanski L., Chiapello E. (1999). Le nouvel esprit du capitalisme. Paris, Gallimard coll. NRF Essais, 843 p.

Boudy J.-F. (2009). Vivre de deux métiers, la pluriactivité. Paris, l'Harmattan, 302 p.

Boutinet J.-P. (2002). Des compétences au projet, un dilemme à prendre en compte pour s'orienter. Carriérologie, vol. 8, $\mathrm{n}^{\circ} 3$, p. 619-633.

Casaux L. (1993). La pluriactivité ou l'exercice par une même personne de plusieurs activités professionnelles. Paris, Librairie générale de droit et jurisprudence, 404 p.

Debaise D. (2005). Expérimentez, n'interprétez jamais. Multitudes, vol. $4, \mathrm{n}^{\circ} 23$, p. 97-100.

Gaillard C., Sourisseau J.-M. (2009). Système de culture, système d'activité(s) et rural livelihood : enseignements issus d'une étude sur l'agriculture kanak (Nouvelle-Calédonie). Le Journal de la Société des Océanistes, vol. 2, n ${ }^{\circ} 129$, p. 279-294.
Gumuchian H., Pecqueur B. (éd.) (2007). La ressource territoriale. Paris, Economica, Anthropos, $252 \mathrm{p}$.

Jaillet M.-C. (2002). De la généralisation de l'injonction au projet. EMPAN, $\mathrm{n}^{\circ} 45$, p. 19-24.

Laurent C., Mundler P. (2006). L'accompagnement de la pluriactivité en question. Résultat de recherche du programme PSDR 2 Territoires, acteurs et agriculture en Rhône-Alpes, 4 p.

Leclerc-Olive M., Engrand S. (2000). Sortir de la précarité par l'emploi : entre routine et projet. In Billard I., Debordeaux D., Lurol M. (éds.), «Vivre la précarité, trajectoires et projets de vie. La Tour d'Aigues: Les éditions de l'Aube ». p. 37-60.

Lenain M.-A., Mamdy J.-F., Rieutort L. (2009). Les très petites entreprises inventives d'entrepreneurs migrants et ancrage territorial : positionnement de recherche. XLVIe colloque de l'ASRDLF - ClermontFerrand, 6, 7, 8 juillet, $16 \mathrm{p}$.

Mairie-Conseils (2005). Synthèse des résultats de l'enquête Mairie-Conseils 2004 sur «Les nouveaux habitants des territoires ruraux ». En Direct de Mairieconseils, $\mathrm{n}^{\circ} 174,4 \mathrm{p}$. 
Nicolas-Le-Strat P. (2007). Expérimentations politiques. Montpellier, Fulenn, 105 p.

Paul J.-L., Bory A., Bellande A. (1994). Quel système de référence pour la prise en compte de la rationalité de l'agriculteur : du système de production agricole au système d'activité. Les cahiers de la recherche-développement, vol. 39, p. 7-19.

Paul M. (2004). L'accompagnement : une posture professionnelle spécifique. Paris, L'Harmattan, $352 \mathrm{p}$.

Perret J. (1999). Tourisme et pluriactivité. Les Cahiers de l'Espace Europe, $\mathrm{n}^{\circ} 15$, p. 87-90.

Saleilles S. (2007). L'entrepreneur néorural et son réseau personnel: une étude exploratoire. Thèse de doctorat, université Montpellier I, $358 \mathrm{p}$.

Tallon H. (2005). La pluriactivité dans le domaine de l'agriculture de l'environnement du tourisme et du patrimoine, source de dynamisme de l'activité et de l'emploi rural du Haut Languedoc. Thèse de master, Montpellier, CIHEAM/IAMM, $167 \mathrm{p}$ Tallon H., Dérioz P., Valette E. (2009). Les projets pluriactifs en zone rurale, entre ancrage territorial et mobilité spatiale ou professionnelle. Le cas des "Hauts Cantons » du département de l'Hérault. XLVIe colloque de l'ASRDLF - ClermontFerrand, 6, 7, 8 juillet, $18 \mathrm{p}$.

Tallon H. (2011). Pluriactivité et accompagnement, un territoire à l'épreuve; une expérimentation par la formation menée dans le Haut Languedoc (Hérault, France). Thèse de doctorat de géographie. Montpellier, Université Paul ValéryMontpellier III. $570 \mathrm{p}$. 